\title{
SPACEMan: Wireless SoC for Concurrent Potentiometry and Amperometry
}

\author{
Daryl $\mathrm{Ma}^{\star}$, Yiyang Chen ${ }^{\star}$, Sara S. Ghoreishizadeh ${ }^{\dagger}$, Pantelis Georgiou ${ }^{\star}$ \\ ${ }^{\star}$ Dept. of Electrical \& Electronic Eng., Centre for Bio-Inspired Technology, Imperial College London, UK \\ ${ }^{\dagger}$ Aspire Centre for Rehabilitation and Assitive Technology, University College London, UK \\ Email: \{darylma, yiyang.chen19, pantelis\}@imperial.ac.uk, s.ghoreishizadeh@ucl.ac.uk
}

\begin{abstract}
This work describes the implementation of SPACEMan, a wireless electrochemical system with concurrent potentiometric and amperometric sensing that can be utilised for saliva, sweat or point of care diagnostics. This system is designed with the vision of simpler interfaces for biofluid analysis. With a complete system-on-chip including electrochemical sensing, power management and data transmission, conventional interfaces like wirebonds will no longer be required in post-processing steps. The proposed architecture consists of a sensor front-end with four electrodes for concurrent amperometric and potentiometric sensing. This front-end outputs square wave signals mixed together with varying frequencies dependent on the sensed input, with the output type switchable with a state machine. A power management system consisting of a low dropout regulator (LDO) band gap reference (BGR), and a rectifier bridge is utilised for supplying power from an inductive link at 433MHz. Sensor data is transmitted wirelessly to a base station using LSK (Load-Shift Keying). The sensor front-end consumes $18 \mu \mathrm{W}$, which the power management system more than adequately provides. The core area of the electronics without the coil is a conservative size of $0.41 \mathrm{~mm}^{2}$.
\end{abstract}

\section{INTRODUCTION}

The development of monolithic wireless electrochemical sensors has quickly gathered pace in recent years [1]-[3]. With the advent of wearables such as the Apple watch and fitbit, a commercially-led push has begun a drive for miniaturised wireless biosensors.

Notable recent examples of wireless electrochemical biosensors have been demonstrated. Kim et al. [4] designed a non-invasive alcohol monitoring wearable tattoo designed for alcoholics. This is detected from sweat through the use of the alcohol-oxidase enzyme. Koehne et al. [5] developed a carbon nanofibre (CNF) electrode array on a silicon wafer for use as a neurotransmitter for dopamine detection. Abrar et al. [6] created a NFC lactate sensor for the continuous measurement of sweat. Kim et al. [7] produced a mouthguard designed for monitoring salivary uric acid levels in real time. Lastly, Douthwaite et al. [8] created a thermally powered ISFET array utilised for $\mathrm{pH}$ measurements in sweat.

The versatility of analyte detection along with a selection of wireless transmission methods are demonstrated in their research. These applications also vary from neural implants to salivary or sweat sensing. A key aspect of this work is to design a miniaturised wireless electrochemical sensor with the versatility and adaptability for multiple purposes.

Having the ability to concurrently utilise potentiometry and amperometry opens up new possibilities. For example, an existing issue with the presence of varying $\mathrm{pH}$ in saliva affects the accuracy of electrochemical sensors [9]. Here, the utilisation of concurrent measurements allows the sensor to be calibrated in real-time [10]. Both of these measurements also have certain advantages and disadvantages [11] - amperometry has a faster detection of glucose in flow cells, while potentiometric technique allows a lower limit of detection, amongst other core differences. Having both techniques maintains the versatility of analyte detection desired for this work. The feasibility of concurrent potentiometry and amperometry sensing using a 4electrode electrochemical cell is demonstrated in our previous work [12].

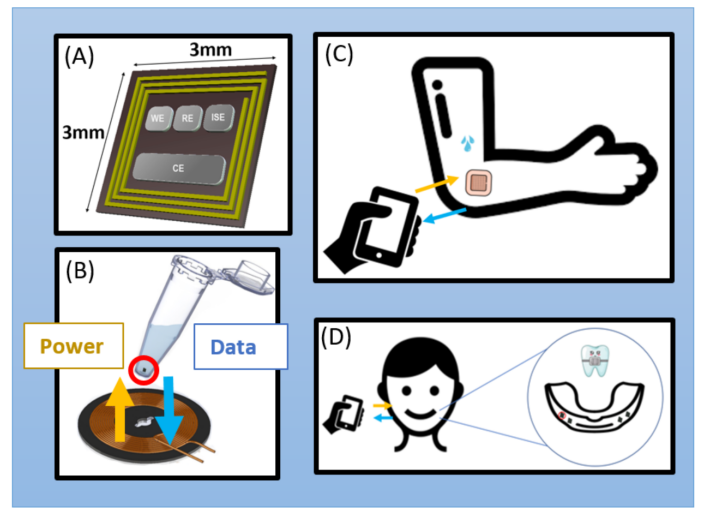

Fig. 1: Concept of a monolithic wireless electrochemical sensor with multiple applications. (A) describes the size of the system developed in this work, with 4 on-chip electrodes. (B) describes the use case of this sensor in a simple sample solution with an external transmitter. (C) describes the sensor embedded in a skin patch for sweat sensing, and (D) describes the sensors embedded in a mouth patch for saliva sensing.

We present an integrated and wireless circuit that enables Simultaneous Potentiometric and AmperometriC mEasureMents (SPACEMan). Fig. 1 illustrates the desired application for the envisaged concept. A system-on-chip, $3 \times 3 \mathrm{~mm}$ sized, for multiple applications in sweat and saliva analysis or point-of-care diagnostics amongst others. The system consists of two key areas: (1) A sensor front end system designed for concurrent amperometry and potentiometry; Here we present an improved design for the front end system based on DAPPER - a low power dual amperometric and potentiometric system [13]. (2) A power and data management system for obtaining power from an external transmitter, along with data transmission. This work features a novel method for downlink through the resetting of the power supply - this reconfigures the circuit to switch in one of four possible states: 


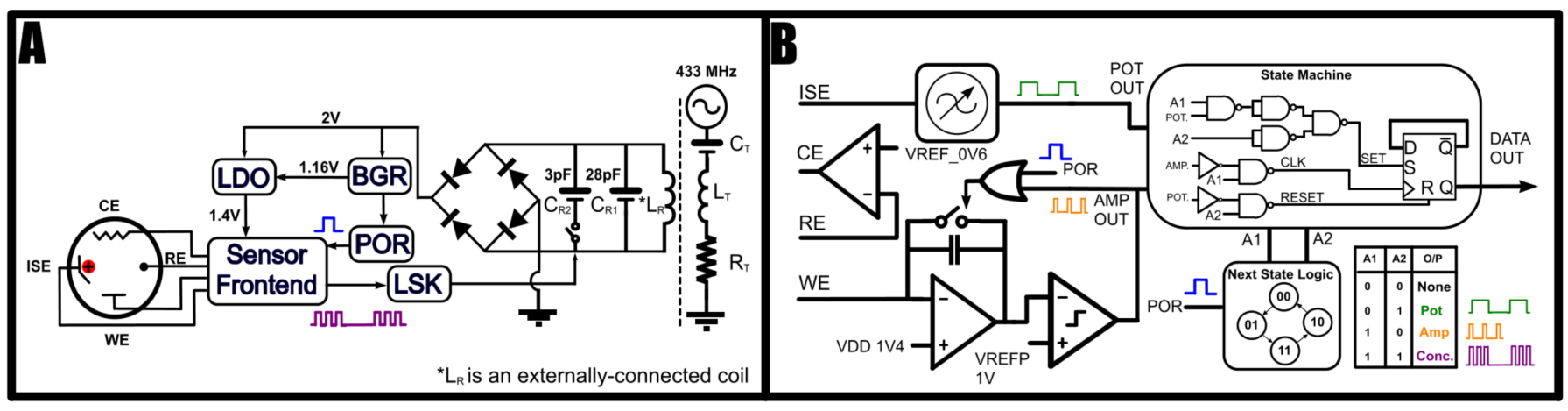

Fig. 2: (2A) System Architecture of SPACEMan, with four electrodes, a sensor frontend, a power management system to supply power wirelessly with a data transmission system for transmitting sensed data. $L_{R}$ is an external coil not included in SPACEMan's architecture.(2B) DAPPER system architecture for dual amperometric and potentiometric sensing. A state machine implements the possibility of outputting either single potentiometry, amperometry, or concurrent sensing.

amperometry measurement only, potentiometry measurement only, concurrently, or none.

This paper is organised as such: Section II describes the system architecture along with the circuit blocks, while Section III describes the implementation, with Section IV describing the simulation results. Section V concludes this work.

\section{System ARChitecture}

Fig. 2A describes the overall system architecture of SPACEMan. A front-end sensor is designed for concurrent sensing with a shared reference electrode. This front-end sensor utilises four electrodes - an ion-sensitive (ISE), counter (CE), working (WE), and reference (RE) electrodes [13]. It transduces a varying frequency from the sensed current and voltage values. These frequencies are then mixed with a D flip-flop, before being transmitted via a load-shift keying circuit at the receiver coil at $C_{R 2}$.

A resonant LC tank previously validated and designed by Feng et al. [14] is used to provide power to SPACEMan. This resonant LC tank consists of an $3 \times 3 \mathrm{~mm}$ on-chip receiver coil $L_{R}$ and a capacitor $C_{R 1}$ that converts the received power from a $433 \mathrm{MHz}$ source to an AC voltage. The frequency is chosen for optimal power delivery and specific absorption rate. This is then converted into a DC voltage by a rectifier circuit. This voltage is used to supply the BGR, which generates a stable reference, and the LDO itself - which generates a supply voltage for the sensor frontend. Upon receiving power from the BGR, the power-on-reset (POR) circuit wakes SPACEMan up and begins the process of transmitting sensor data through to the LSK circuit. To communicate the change of state to SPACEMan, a pause in the transmission is utilised to trigger the POR circuit for switching states. This allows the output of the system to be switched between 4 different possible states.

\section{IMPLEMENTATION}

SPACEMan is implemented in TSMC180nm technology with the system broken down to 4 key modules described in this section.

\section{A. Amperometric and Potentiometric Sensing Front End}

The overall system along with the transmitted signals is shown in Fig. 2B. The front end consists of five main blocks: (1) A potentiometric circuit consisting of a voltage controlled oscillator and a voltage buffer; (2) An amperometric circuit consisting of a switched-capacitor integrator and a comparator in a feedback loop; (3) A state machine with a SR D-flipflop for mixing the signals together triggered by the POR; (4) Amplifiers utilised for supplying the counter and the reference electrodes; (5) A POR circuit for starting up the system/changing states when power is received.

The principle of operation is as follows: An amplifier in negative feedback drives $\mathrm{CE}$ and $\mathrm{RE}$ with a reference voltage. This provides the reference required for both the potentiometric and amperometric circuits. The sensed voltage on the ISE is detected by the potentiometric circuit. A range of input voltage from $0.3 \mathrm{~V}$ to $1 \mathrm{~V}$ is converted into a frequency range of $1-9 \mathrm{~Hz}$. At the same time, the sensed current input from the WE is detected by the amperometric circuit for an input range of $80 \mathrm{pA}$ to $1 \mu \mathrm{A}$. The switched capacitor integrator and the comparator in a feedback loop converts this current into a frequency range of $400 \mathrm{~Hz}-400 \mathrm{kHz}$. This circuit is an optimised form of [13] with a lower power consumption of $62 \%$ lower power at the cost of reducing input current range from the previous $250 \mathrm{pA}-5.6 \mu \mathrm{A}$ range to $80 \mathrm{pA}-1 \mu \mathrm{A}$.

\section{B. State Machine and Downlink Communication}

The introduction of a state machine is utilised to externally control the outputs of the sensor front-end. This allows the system to be switched with a pulse generated from the POR, and allows the user to switch the output states of the circuit. The truth table shown in Fig. 2B describes the output bits for each state. Bits A1 and A2 set the type of output to be sent to the D flip-flop with set-reset inputs. Based on the set state, the input to the LSK will be (1) POT OUT, (2) AMP OUT halved, or (3) AMP OUT modulated by POT OUT.

The frequencies of signals POT OUT and AMP OUT are chosen to be at least a magnitude of $20 \times$ apart to reduce any errors from mixing the signals. This also allows the user 


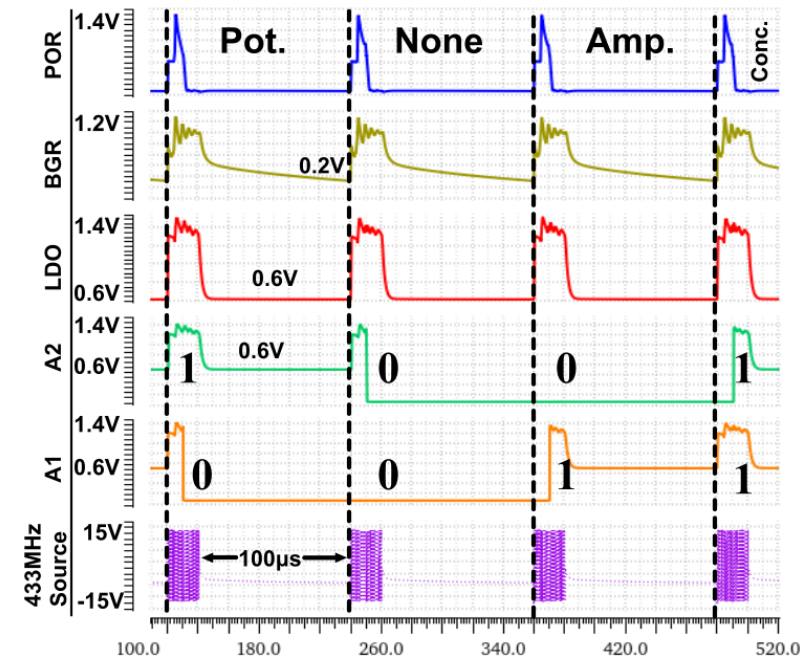

Fig. 3: Post-layout simulation of how the states are triggered. A $100 \mu$ s pause in the transmission of the $433 \mathrm{MHz}$ source causes the LDO to drop to 0.6V due to the presence of $\mathrm{C}_{R 1}$, that maintains a voltage at the output. The BGR which triggers the POR decreases to $0.2 \mathrm{~V}$. When the transmission begins again, the logic high on $\mathrm{A} 1$ and $\mathrm{A} 2$ goes back up to $1.4 \mathrm{~V}$ just before the POR pulse triggers the next state.

to easily determine the output state of the circuit by looking at the range of frequencies at the LSK. This signal is then transmitted via LSK through to a base-station.

The states are triggered through turning the energy source off for $100 \mu \mathrm{s}$ as shown in Fig. 3. The POR pulses are clocked into a latched logic circuit that changes bits A1 and A2 for varying the states. (1) When the source is off, a $100 \mu \mathrm{s}$ pause causes the BGR voltage to drop to $0.2 \mathrm{~V}$. The LDO voltage which powers the circuits goes down to $0.6 \mathrm{~V}$, which is followed by the logic bits A1 and A2 if they are in a high state. (2) When the source is switched on, the LDO's fast response time of 100ns ensures that the bits $\mathrm{A} 1$ and $\mathrm{A} 2$ go up to their logic high voltage of $1.4 \mathrm{~V}$. The BGR goes up to $1.2 \mathrm{~V}$ moments later, triggering the POR pulse to go to $1.4 \mathrm{~V}$. This triggers the logic state to switch dependent on the output code. If the circuit is held off for more than $1.47 \mathrm{~ms}$, bits A1 and A2 will revert back to logic lows. At that point in time, the charge on $\mathrm{C}_{R 1}$ is lost - resulting in the logic circuits not retaining the information on the previous state. The next state logic is coded using gray encoding for a simpler state machine, with only an inverter and $2 \mathrm{D}$ flip-flops required for the latching.

Multiple inputs and single channel output systems conventionally utilise downlink techniques such as code-division multiple access (CDMA) or frequency-division multiple access (FDMA). These methods either require decoders or phaselocked loop circuits, which are power intensive. The chosen method employed in this work uses significantly less power due to its simplicity of implementation. However, this chosen method comes with several limitations, namely: (1) The timing of the pauses has to be calculated accurately. Too long, and the state logic circuit will not change. Too short, and the POR pulse will not be high enough to trigger a state change. (2) The circuit is susceptible to process variation. As the timing is key, it is important the POR pulse is triggered when the VDD is back to the normal state.

A POR circuit is applied to provide a pulse for DAPPER to start, as well as trigger the state change circuit. Lastly, stable reference voltages are generated through the use of potential dividers from the $1.4 \mathrm{~V}$ supply.

\section{Power Management}

The power management system consists of 3 main blocks: A full-wave bridge rectifier, a high power supply rejection ratio (PSRR) BGR, and a LDO. The bridge rectifier serves the purpose of providing a $2 \mathrm{~V}$ DC supply voltage from the 433 MHz signal. This is implemented with 4 Schottky diodes with a simulated power transfer efficiency (PTE) of $60 \%$. This was chosen above other topologies such as the cross-coupled, diode-connected, and boot-strapping to prioritise PTE.

The BGR circuit has the requirement of supplying a stable reference voltage in the presence of a high supply voltage fluctuation $(2.05 \mathrm{~V}-2.1 \mathrm{~V})$ caused by the LSK. The design of the BGR circuit is inspired by [15]. It achieves a high PSRR of $-60 \mathrm{~dB}$ at $100 \mathrm{kHz}$, with a $1 \mathrm{mV}$ output voltage deviation.

The LDO circuit down-converts the $2 \mathrm{~V}$ from the rectifier to $1.4 \mathrm{~V}$ for the front end circuit.

\section{Data Management}

The data transmission scheme is chosen as LSK above other known methods due to its simplicity, low area and relatively high power efficiency. The principle behind this circuit is based on shifting the resonant frequency of the LC tank. This can be easily implemented by shorting a parallel capacitor $C_{R 2}$. However, it is important to note the effects of the size of $C_{R 2}$ on the system. If $C_{R 2}$ is too large, the supply voltage ripple will be high - which lowers the PTE of the system. On the other hand, if $C_{R 2}$ is too low, the impedance change will not be high enough to be observed on the transmitter side. A trade-off is chosen between power efficiency and voltage shift.

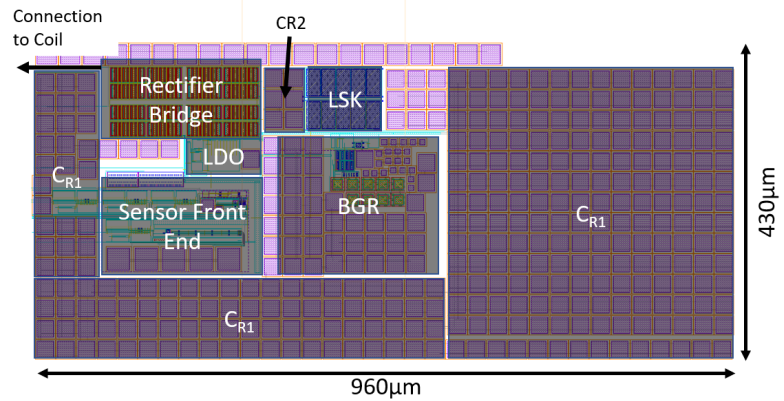

Fig. 4: Layout of SPACEMan presenting the BGR, LSK, LDO, Rectifier Bridge and Sensor Front End. Note the relative size of $C_{R 1}$ compared to the rest of the electronics.

\section{Simulation Results}

The circuit was designed and simulated on commercially available TSMC 180nm technology. The overall layout presented in Fig. 4 shows the core system area of $0.41 \mathrm{~mm}^{2}$. 


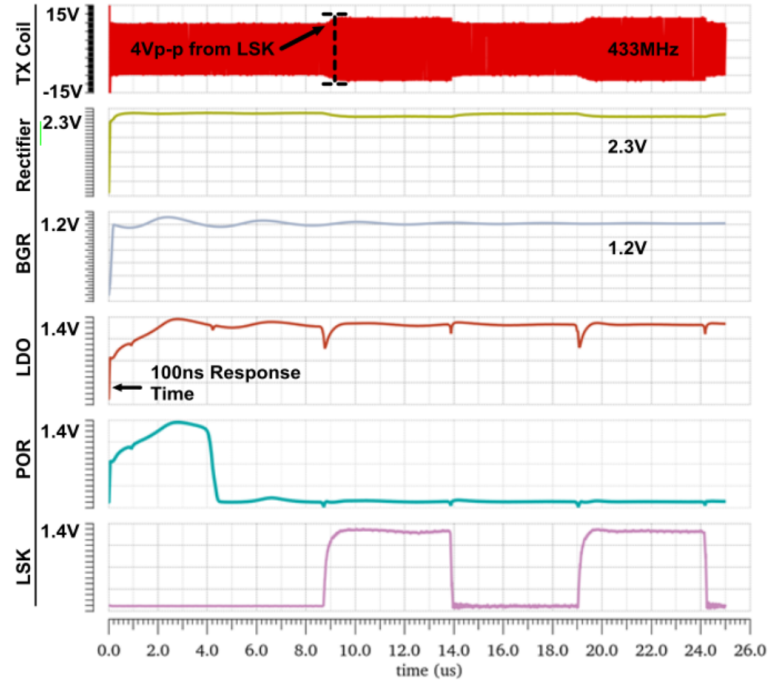

Fig. 5: Timing Diagram of the first $26 \mu$ s upon startup showing the most important voltages in the power management circuit at startup. The transmitter coil is driven by a $3 \mathrm{~V}_{p-p}$ sinewave at $400 \mathrm{kHz}$ using a voltage boost and power amplifier previously published in [14]. The coupling factor of the inductive link was set to 0.216 in the simulations in line with [14].

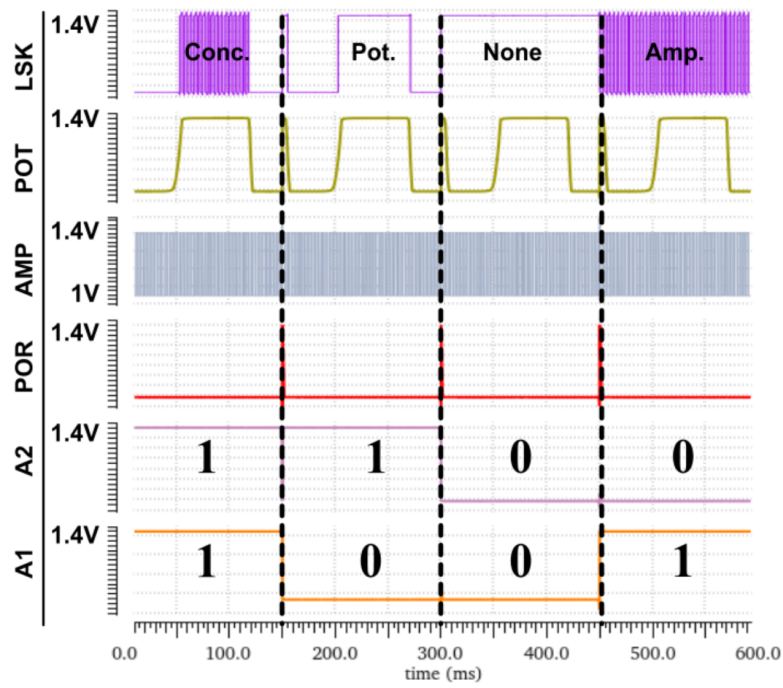

Fig. 6: Simulated voltage levels at the input and output of the state machine from start up to $t=600 \mathrm{~ms}$. The following test inputs are used in the simulation setup: input current to amperometry circuit: $80 \mathrm{pA}$. Input voltage to potentiometric circuit: $0.3 \mathrm{~V}$. The inductive link connection of the power management circuit is bypassed and instead an ideal voltage source $(2 \mathrm{~V})$ is used to power on the LDO, BGR, POR, LSK and Sensor Frontend circuits to speed up the simulations.

The amperometric circuit has a linear range for an input of $80 \mathrm{pA}$ to $1 \mu \mathrm{A}$, with an output frequency of $400 \mathrm{~Hz} @ 80 \mathrm{pA}$ to $2.4 \mathrm{kHz} @ 1 \mu \mathrm{A}$. The circuit draws a maximum power of $10.4 \mu \mathrm{W} @ 400 \mathrm{kHz}$. The potentiometric circuit has an input of $0.3-1 \mathrm{~V}$ based on the possible voltages measured with an IrOx electrode [16]. The linear range of the circuit ranges from $9.27 \mathrm{~Hz} @ 0.3 \mathrm{~V}$ to $1.51 \mathrm{~Hz} @ 1 \mathrm{~V}$. This circuit has a maximum power consumption of $4 \mu \mathrm{W} @ 9.27 \mathrm{~Hz}$.

Fig. 5 describes the process on startup. The Tx coil
TABLE I: Comparison with state-of-the-art

\begin{tabular}{|c|c|c|c|}
\hline Paper & This Work & [17] & [18] \\
\hline Technology (nm) & 180 & 65 & 350 \\
\hline Supply (V) & 1.4 & 0.9 & 1.4 \\
\hline Power $(\mu \mathbf{W})$ & 18 & 0.97 & 92 \\
\hline Dynamic Range (dB) & $\begin{array}{c}62 \text { (pot.) } \\
87 \text { (amp.) }\end{array}$ & $\begin{array}{c}\text { 43(pot.) } \\
30.1 \text { (amp.) }\end{array}$ & 66 (pot.) \\
\hline Size $\left(\mathbf{m m}^{2}\right)$ & 0.41 & 1.275 & 2.1 \\
\hline Uplink Type & LSK & LSK & LSK \\
\hline Downlink Type & POR & NA & NA \\
\hline Trans. Mech. & Amp. \& Pot. & Amp. \& Pot. & Pot. \\
\hline Data Rate & $400 \mathrm{kHz}$ & NA & $825 \mathrm{~Hz}$ \\
\hline
\end{tabular}

demonstrates the transmitted $433 \mathrm{MHz}$ signal used to power SPACEMan. The transmitted LSK data can be seen as an additional $4 \mathrm{~V}_{p-p}$ modulation on the transmitted $433 \mathrm{MHz}$ signal. This modulated voltage is used to decode the LSK data on the transmitter side. The rectifier transmits a stable $2.3 \mathrm{~V}$ supply, along with the BGR at $1.2 \mathrm{~V}$. The LDO maintains a stable $1.4 \mathrm{~V}$ supply, although ripples occur at points where the front end sensor transmits a high pulse. The POR circuit showcases a pulse upon startup. This starts up the circuit after a propagation delay of roughly $3.5 \mu \mathrm{s}$, after which the frontend system begins transmitting the output data through to the LSK circuit.

Fig. 6 showcases the input/output characteristics of the state machine up to $600 \mathrm{~ms}$. The amperometric and potentiometric circuits transduce their frequencies based on a simulated input of $0.3 \mathrm{~V}(9 \mathrm{~Hz})$ and $80 \mathrm{pA}(400 \mathrm{~Hz})$. The inductive link connection is bypassed to provide an ideal $2 \mathrm{~V}$ source to power on the LDO, BGR, POR, LSK and Sensor Frontend to speed up the simulation. The 4 possible states are described in the figure.

Table. I compares the system characteristics to other SoC systems. The small size of the core electronics, as well as the simplicity of application makes it particularly versatile compared to other work.

\section{CONCLUSION}

This work describes a miniaturised low powered system-onchip for wireless sensing of potentiometry and amperometry. This system is able to do both sensing techniques at a low power, while transmitting the data wirelessly to a base station. The core focus of this system prioritises the sensor front end, which operates at a low power of $18 \mu \mathrm{W}$. A novel downlink system is used to switch the output states, along with a power and data management system for powering the circuits. The versatility and adaptability of this chip makes it a particularly valuable asset in future implementations of wearable devices.

The next immediate step is to simulate the effect of process, voltage and temperature (PVT) variations on the performance of the AFE and the BGR of SPACEMan before being sent for fabrication. Moving forward, the system will be connected to an external coil to further test the functionality of the wireless power and data management systems. Finally, SPACEMan will be coupled to electrode biosensors for the applications mentioned in Fig. 1. 


\section{REFERENCES}

[1] S. Shrivastava, T. Q. Trung, and N.-E. Lee, "Recent progress, challenges, and prospects of fully integrated mobile and wearable point-of-care testing systems for self-testing," Chemical Society Reviews, vol. 49, no. 6, pp. 1812-1866, 2020.

[2] H. Lyu, J. Wang, J. La, J. M. Chung, and A. Babakhani, "An energyefficient wirelessly powered millimeter-scale neurostimulator implant based on systematic codesign of an inductive loop antenna and a custom rectifier," IEEE Transactions on Biomedical Circuits and Systems, vol. 12, no. 5, pp. 1131-1143, 2018.

[3] H. Kim, H. Hirayama, S. Kim, K. J. Han, R. Zhang, and J. Choi, "Review of near-field wireless power and communication for biomedical applications," IEEE Access, vol. 5, pp. 21 264-21 285, 2017.

[4] J. Kim, I. Jeerapan, S. Imani, T. N. Cho, A. Bandodkar, S. Cinti, P. P. Mercier, and J. Wang, "Noninvasive alcohol monitoring using a wearable tattoo-based iontophoretic-biosensing system," Acs Sensors, vol. 1, no. 8, pp. 1011-1019, 2016.

[5] J. E. Koehne, M. Marsh, A. Boakye, B. Douglas, I. Y. Kim, S.-Y. Chang, D.-P. Jang, K. E. Bennet, C. Kimble, R. Andrews et al., "Carbon nanofiber electrode array for electrochemical detection of dopamine using fast scan cyclic voltammetry," Analyst, vol. 136, no. 9, pp. 1802$1805,2011$.

[6] M. A. Abrar, Y. Dong, P. K. Lee, and W. S. Kim, "Bendable electrochemical lactate sensor printed with silver nano-particles," Scientific reports, vol. 6, p. 30565, 2016.

[7] J. Kim, S. Imani, W. R. de Araujo, J. Warchall, G. Valdés-Ramírez, T. R. Paixão, P. P. Mercier, and J. Wang, "Wearable salivary uric acid mouthguard biosensor with integrated wireless electronics," Biosensors and Bioelectronics, vol. 74, pp. 1061-1068, 2015.

[8] M. Douthwaite, E. Koutsos, D. C. Yates, P. D. Mitcheson, and P. Georgiou, "A thermally powered isfet array for on-body ph measurement," IEEE Transactions on Biomedical Circuits and Systems, vol. 11, no. 6 , pp. 1324-1334, 2017.

[9] A. J. Bard and L. R. Faulkner, Electrochemical methods : fundamentals and applications / Allen J. Bard, Larry R. Faulkner. Wiley New York, 1980.

[10] M. J. Schöning, R. Krause, K. Block, M. Musahmeh, A. Mulchandani, and J. Wang, "A dual amperometric/potentiometric fia-based biosensor for the distinctive detection of organophosphorus pesticides," Sensors and Actuators B: Chemical, vol. 95, no. 1-3, pp. 291-296, 2003.

[11] K. Brunt, "Comparison between the performance of an electrochemical detector flow cell in a potentiometric and an amperometric measuring system using glucose as a test compound," Analyst, vol. 107, no. 1279, pp. 1261-1271, 1982.

[12] D. Ma, S. S. Ghoreishizadeh, and P. Georgiou, "Concurrent potentiometric and amperometricsensing with shared reference electrodes," IEEE Sensors Journal, 2020.

[13] D. Ma, S. S. Ghoreishizadeh, and P. Georgiou, "Dapper: A low power, dual amperometric and potentiometric single-channel front end," in 2020 IEEE International Symposium on Circuits and Systems (ISCAS), 2020, pp. $1-5$.

[14] P. Feng, "Completely wireless infrastructure for distributed mm-sized neural implants," PhD Thesis, 2020.

[15] W. Li, R. Yao, and L. Guo, "A low power cmos bandgap voltage reference with enhanced power supply rejection," in 2009 IEEE 8th International Conference on ASIC. IEEE, 2009, pp. 300-304.

[16] D. Ma, C. Mason, and S. Ghoreishizadeh, "A wireless system for continuous in-mouth ph monitoring," in 2017 IEEE Biomedical Circuits and Systems Conference, BioCAS 2017-Proceedings. IEEE, 2018.

[17] H. Jiang, X. Zhou, S. Kulkarni, M. Uranian, R. Seenivasan, and D. A. Hall, "A sub-1 $\mu \mathrm{w}$ multiparameter injectable biomote for continuous alcohol monitoring," in 2018 IEEE Custom Integrated Circuits Conference (CICC). IEEE, 2018, pp. 1-4.

[18] L. B. Leene, M. Maslik, P. Feng, K. M. Szostak, F. Mazza, and T. G. Constandinou, "Autonomous soc for neural local field potential recording in mm-scale wireless implants," in 2018 IEEE International Symposium on Circuits and Systems (ISCAS). IEEE, 2018, pp. 1-5. 